\title{
Two-Photon Direct Laser Writing for Proteinaceous Microstructures with Additional Sensitizer
}

\author{
Daniela Serien*,**, and Shoji Takeuchi*,** \\ * The University of Tokyo, Institute of Industrial Science (IIS), 4-6-1 Komaba, Meguro-ku, Tokyo \\ 153-8505, Japan; main author correspondence: serien@iis.u-tokyo.ac.jp \\ ** ERATO Takeuchi Biohybrid Innovation Project, Japan Science and Technology Agency, 4-6-1 \\ Komaba, Meguro-ku, Tokyo, 153-8505, Japan
}

We report our findings of proteinaceous microstructures polymerized by two-photon direct laser writing (DLW) using an additional sensitizer for rapid fabrication. Conventionally, a solution of protein and one type of photosensitizer or photo-initiator is exposed to laser light for protein cross-linking. We introduce an additional photosensitizer of different absorption properties and potential for radicalization to create a three-component photosensitizing system. The advantage of a three-component system is an enhancement of photopolymerization performance. Due to the enhancement, rapid polymerization is achieved without increasing the amount of available monomer in pre-polymer solution. Depending on the target protein, obtaining sufficient amounts of protein might require financial or purification effort. Therefore, a three-component photosensitizing system is promising to avoid raising protein concentration in solution. We characterize the photosensitizing system based on a previously introduced fitting model and measurement method; thickness and height of fully restrained beam microstructures are analyzed by scanning electron microscopy in dependency of the applied laser power and exposure time. We compare the fit-obtained results of two three-component proteinaceous pre-polymer solutions with a previously introduced two-component system to elaborate on the application of the model. Proteinaceous threecomponent photosensitizing systems enable rapid fabrication simultaneously with a reduction of protein concentration in solution.

DOI: 10.2961/jlmn.2017.02.0006

Keywords: laser direct writing (LDW), photoinitiating system (PIS), bovine serum albumin (BSA), protein cross-linking, three-component

\section{Introduction}

Amongst light-initiated 3D printing technologies, direct laser writing (DLW) is particularly interesting because the confinement of photo-initiated polymerization to the laser focal volume enables high precision and high resolution [1]. For conventional photoresists, DLW fabrication has been advanced to enable fabrication of mesoscale-sized structures with DLW-associated submicron-sized features [2].

Considering DLW-facilitated photo-initiation of protein cross-linking [3,4] for biocompatible microstructures, fabrication of proteinaceous microstructures has been applied to $\mathrm{pH}$-responsive microdevices such as tunable lenses [5] and shape-shifting microstructures [6,7]. Optical elements constructed by biomaterial are considered relevant for ecocompatible optics; optics that are disposable without impacting natural environment [8]. Furthermore, because the crosslinked proteins maintain their protein binding affinity $[9,10]$, biomedical applications such as microfluidic devices for onsite detection [10] might be facilitated. However, fabrication of microstructure arrays or mesoscale structures currently requires several hours to days. Although an increase of photosensitizer concentration in the pre-polymer solution might increase fabrication speed, previous reports show that a) Conventional: two components b) Here: three components

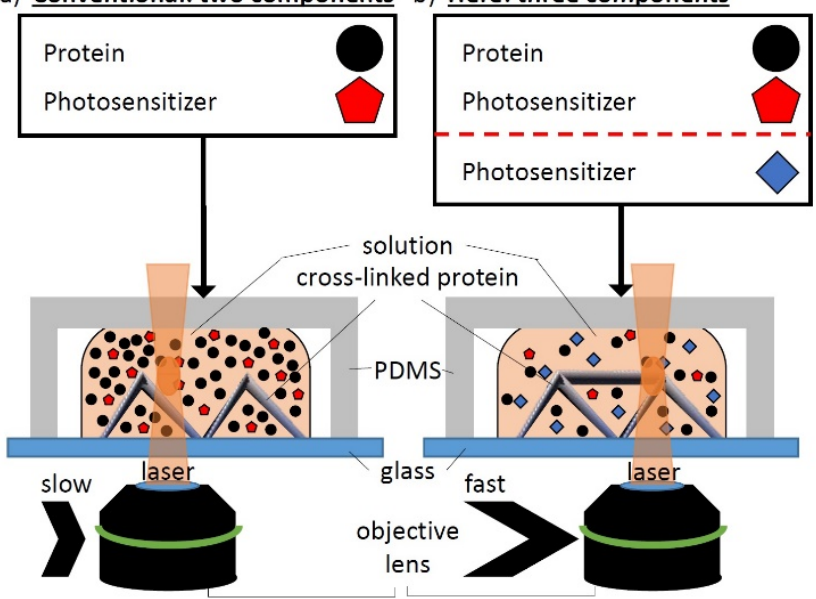

Figure 1 Introduction of an additional photosensitizer Concept of direct laser writing of a free-standing, fully restrained beam structure within protein pre-polymer solution of a) conventional two components and b) here proposed three components.

only a single type of photosensitizer induces a quick saturation of polymerization [4,11]. 
Here, we report the use of a three-component photosensitizing system for rapid fabrication of proteinaceous microstructures by DLW (Figure $1 \mathrm{a}, \mathrm{b})$. Three-component photoinitiating or photosensitizing systems utilize two photoinitiators or photosensitizers for the polymerization of a monomer system to enhance photosensitivity and photopolymerization performance [12,13]. We add photosensitizer rhodamine B (RhB) with its different absorption properties [14] and potential for radicalization [15] to the established system of photosensitizer and protein [3-11]. Specifically, we chose to add $\mathrm{RhB}$ to a system of photosensitizer flavin adenine dinucleotide (FAD), which is suitable for in situ fabrication [16], and protein bovine serum albumin (BSA), which is a commonly used protein for crosslinking [4-11]. Based on a previously introduced protein polymerization model [11, see Appendix], we analyze and compare three-component photosensitizing systems regarding polymerization conditions and feature sizes. We show that for a three-component photosensitizing system fabrication speed increases while the protein monomer in solution is reduced. Such reduction of the required protein quantities in solution might be interesting for fabrication with proteins that are considerably less abundant than BSA. As exemplary use of a considerably less abundant protein such as cell adhesion protein collagen, we introduce collagen type-IV to the threecomponent proteinaceous photosensitizing system.

\section{Experimental section}

\subsection{Fabrication of proteinaceous microstructures}

Proteinaceous microstructures were fabricated by dropcasting 3-4 $\mu \mathrm{L}$ of proteinaceous pre-polymer solution on cleaned cover glasses (CO13001, Matsunami Glass Ind., Ltd., Japan). Fs-pulsed $780 \mathrm{~nm}$ laser light was exposed via a 100xobjective lens with N.A. $=1.4$ by Photonic Professional (Nanoscribe, Germany) (Figure 1 a,b). The system is equipped with a FemtoFiberPro 2099 NIR laser (Toptica, Germany) that generates a peak power density of approximately $2.125 \mathrm{~mJ} / \mathrm{m}^{2}$ and is polarized over $95 \%$ (horizontal). A chamber of polydimethylsiloxane (PDMS, Silpot 184\&Cat, Dow Corning, USA) covers the solution in order to delay drying. We fabricated arrays of fully constrained beam structures on tetrahedral supports in dependency of laser power (in front of the objective lens) and writings speed, devised as previously reported [11]. Writing speed is an input parameter, called scan speed, to the Photonic Professional in order to control piezo stage movement. After fabrication, the samples were washed well in distilled (MilliQ) water. For scanning electron microscopy (SEM) by SU-8000 (Hitachi, Japan), samples were washed in subsequent steps with ethanol (14033, Kanto chemical co., Inc., Japan) and 1butanol (026-03326, Wako 1S, Pure Chemical Industries, Ltd., Japan), then freeze-dried with ES-2030 (Hitachi, Japan) and sputtered with platinum (MSP- Vacuum Device, Japan). Side view of microstructures at $90^{\circ}$ was achieved by a $30^{\circ}$-tilt of a manufactured $60^{\circ}$-steep acrylic stand.
We introduce proteinaceous pre-polymer solutions as three-component photosensitizing systems: the three components are protein, one photosensitizer and an additional photosensitizer (Figure $1 \mathrm{~b}$ ). Different proteins contribute to the species of protein monomer for cross-linking and are therefore considered as one component to the photosensitizing system. The conventional two-component photosensitizing system (BSA-FAD) consists of $200 \mathrm{mg} / \mathrm{mL}$ bovine serum albumin (BSA, A-7906, Sigma-Aldrich), $1 \mathrm{mM}$ flavin adenine dinucleotide (FAD, F-6625, Sigma-Aldrich) dissolved in $50 \%$ $0.02 \mathrm{M}$ 4-(2-hydroxyethyl)-1-piperazineethanesulfonic acid (HEPES, H-3375, Sigma-Aldrich) prepared at $\mathrm{pH}=7.4$ and $50 \%$ dimethyl sulfoxide (DMSO, 043-07216, Wako Pure Chemical Industries, Ltd., Japan), as reported previously [11]. The three-component system (BSA-FAD-RhB) consists of $50 \mathrm{mg} / \mathrm{mL}$ BSA, $250 \mu \mathrm{M} \mathrm{FAD}$, and $611.3 \mu \mathrm{M}$ rhodamine B (RhB, 183-00122, Wako Pure Chemical Industries, Ltd., Japan) dissolved in $12.5 \% 0.02 \mathrm{M}$ HEPES prepared at $\mathrm{pH}=7.4$, $22.25 \%$ DMSO, $21.75 \%$ methanol (132-06471, Wako Pure Chemical Industries, Ltd., Japan), and $43.5 \%$ acetone (01026, Kanto chemical co., Inc., Japan). Solution of BSA-FAD-RhB with collagen type IV consists of $150 \mu \mathrm{g} / \mathrm{mL}$ collagen typeIV (Cellmatrix Type IV, 638-05921, Nitta Gelatin Inc., Japan), $25 \mathrm{mg} / \mathrm{mL}$ BSA, $125 \mu \mathrm{M}$ FAD, and $305.7 \mu \mathrm{M} \mathrm{RhB}$ dissolved in $45 \% \mathrm{pH}=3$ adjusted MilliQ water, $5 \%$ stabilizer solution of Cellmatrix Type IV, 6.25 \% HEPES, 11.1 \% DMSO, $10.9 \%$ methanol, and $21.75 \%$ acetone. An oxygen scavenger system $[17,18]$ added to these solutions seemingly extended shelf life without influencing polymerization notably.

\subsection{Data analysis}

Measurements were obtained from image analysis by Fiji/ImageJ (U. S. National Institutes of Health, U.S.). All measurements were corrected for the respective average loss of $12 \%, 14 \%$ or $18 \%$ after drying; Due to dehydration during freeze-dry, beam length observed afterwards in SEM was shorter than originally devised beam length of $4 \mu \mathrm{m}$ which was confirmed in liquid by bright field microscopy. When enough data points were available, low and high laser power margin were removed due to apparent instability.

The data sets for thickness and height are evaluated simultaneously as global fit to the previously reported model [11, see Appendix]. $z_{\text {dif } f}$ was changed accordingly to the change of refractive index $n$ of the pre-polymer solution due to organic solvents, such as DMSO [19]. Data is fitted globally, i.e. diameter and height are paired and simultaneously minimized for the goodness of fit value, reduced $\chi^{2}$. Additionally, the coefficient of determination, $\mathrm{R}^{2}$, is calculated. Fitting results are radial scaling factor $\alpha_{R}$ and axial scaling factor $\alpha_{z}$, that describe kinetics of radical chain reaction and diffusion, and $F$ that summarizes parameters of light, absorption and pre-polymer solution. From $F$, we obtain the threshold dose equivalent $D_{t h}=F / r_{\text {diff }}^{4}$. All graphs display average values with standard deviation. Statistical analysis was performed as student $t$-test. 

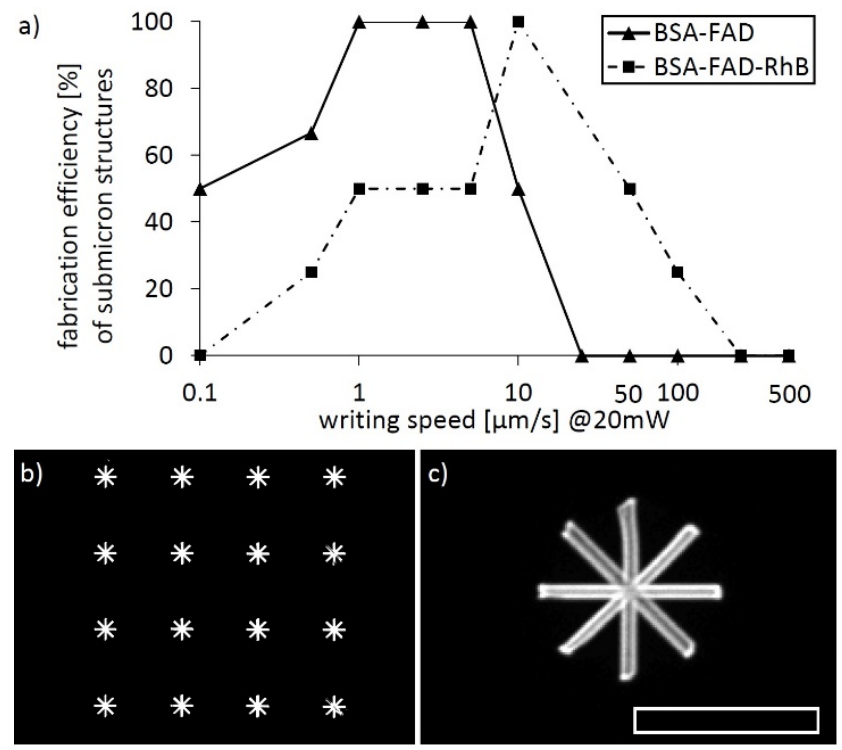

Figure 2 Fabrication efficiency of submicron beam elements at different writing speeds and array fabrication. Writing speed is an input parameter for DLW piezo movement. a) At the laser power of $20 \mathrm{~mW}$, the fabrication with a conventional BSA-FAD system (solid, $n=6$ ) is feasible up to $10 \mu \mathrm{m} / \mathrm{s}$, when fabrication with BSA-FAD-RhB system (dashed, $n=4$ ) is feasible up to $100 \mu \mathrm{m} / \mathrm{s}$. b,c) Confocal laser scanning image of b) an array of flower-shaped microstructures, and c) close-up view of a microstructure. Scale bar represents $15 \mu \mathrm{m}$.

Fabrication efficiency was evaluated regarding the writing speed of obtainable diameter measurement; this evaluation disregards polymerization that did not lead to submicronsized features and disregards incomplete polymerization results close to polymerization threshold.

\section{Results and discussion}

\subsection{Measurement of writing speed}

At first, we investigate the fabrication speed by comparing feasible writing speed of two- and threecomponent photosensitizing systems (Figure 2 a). Using the conventional two-component photosensitizing system BSAFAD, we observed maximal writing speed at the laser power of $20 \mathrm{~mW}$ (in front of the objective lens) to be $10 \mu \mathrm{m} / \mathrm{s}$ fabricating fully restrained beam microstructures. Using the three-component photosensitizing system BSA-FAD-RhB, we observed successful 3D microstructure fabrication at $100 \mu \mathrm{m} / \mathrm{s}$. The peak representing writing speed for high fabrication efficiency shifts from $5 \mu \mathrm{m} / \mathrm{s}$ to $10 \mu \mathrm{m} / \mathrm{s}$ by the introduction of $\mathrm{RhB}$. This result indicates a 2- to 10 -fold increase of fabrication speed.

Fabrication of an array of flower-shaped microstructures was completed within a few minutes utilizing the BSA-FADRhB at laser power of $15 \mathrm{~mW}$ and writing speed $5 \mu \mathrm{m} / \mathrm{s}$ as well as $25 \mu \mathrm{m} / \mathrm{s}$ (Figure $2 \mathrm{~b}, \mathrm{c}$ ). Furthermore, we observed 2D fabrication at a peak writing speed of $1000 \mu \mathrm{m} / \mathrm{s}$ at a laser power of $65 \mathrm{~mW}$. With such rapid fabrication, it became possible to fabricate batches of proteinaceous microstructures within minutes to hours.

In comparison with the current records of $200 \mathrm{~mm} / \mathrm{s}$ for industrial DLW fabrication and $7 \mathrm{~mm} / \mathrm{s}$ for photoresists [21], the increase of writing speed appears marginal. Contrary, fabrication of proteinaceous microstructures has previously been reported with $1-5 \mu \mathrm{m} / \mathrm{s}$ for FAD-BSA systems of similar or higher pre-polymer solution concentrations [9, 16] or recent fabrication with $100 \mu \mathrm{m} / \mathrm{s}$ for 2.5 -fold BSA concentration with methylene blue [8]. The increase of writing speed without increase of pre-polymer constituents is of relevant order of magnitude.

\subsection{Characterization of the three-component photosensitizing system BSA-FAD-RhB}

In order to show that rapid fabrication of BSA-FAD-RhB is systematic and its feature sizes are predictable, we characterize the system of BSA-FAD-RhB. We fit diameter and height of polymerization elements (voxel) (Figure 3 a) with changing fabrication conditions, laser power and exposure time, to a previously introduced model [11].

We observed submicron feature sizes; the smallest feature sizes in our observation were the data pair of thickness of $180 \mathrm{~nm}$ and height of $420 \mathrm{~nm}$ after correction for dehydration loss of $12 \% \pm 5 \%$. The fitting result was a radial scaling factor $\alpha_{R}=0.52 \pm 0.02$, and an axial scaling factor $\alpha_{Z}=0.45 \pm 0.02$ representing complex kinetics of radical chain reaction and diffusion. We furthermore obtained the parameter

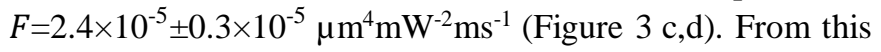
parameter, we conclude that the threshold condition is $D_{t h}=550 \pm 70 \mathrm{~mW}^{2} \mathrm{~ms}$. In comparison with the previously reported threshold condition $D_{t h}=2800 \pm 680 \mathrm{~mW}^{2} \mathrm{~ms}$ of BSAFAD [11], a 3- to 7-fold increase of writing speed is estimated at comparable laser powers.

This characterization indicates that application of the polymerization model with scaling factors is a good measure to predict fabrication feature sizes for the three-component photosensitizing system.

\subsection{Characterization of the three-component} photosensitizing system BSA-FAD-RhB with collagen type-IV

In order to harness different protein functions, appropriate proteins are required to be involved in the cross-linking as monomers. In the following, we demonstrate that microfabrication remains rapid when BSA-FAD-RhB is complemented with protein collagen type-IV diluted in its commercial buffer (Figure 4).We observed submicron feature sizes (Figure $4 \mathrm{a}, \mathrm{b}$ ); The smallest feature sizes in our observation were the data pair of thickness of $150 \mathrm{~nm}$ and height of $120 \mathrm{~nm}$ after correction for dehydration loss of $14 \% \pm 5 \%$. The fitting result was $\alpha_{R}=0.56 \pm 0.04, \alpha_{Z}=0.45 \pm 0.04$, and $F=2.2 \times 10^{-5} \pm 0.3 \times 10^{-5} \mu \mathrm{m}^{4} \mathrm{~mW}^{-2} \mathrm{~ms}^{-1}$ (Figure $4 \mathrm{c}$,d). From this fitting result, we conclude that the threshold condition is $D_{t h}=610 \pm 70 \mathrm{~mW}^{2} \mathrm{~ms}$. This result shows that writing speed is estimated to be increased 3-to 6-fold from BSA-FAD at comparable laser power. 

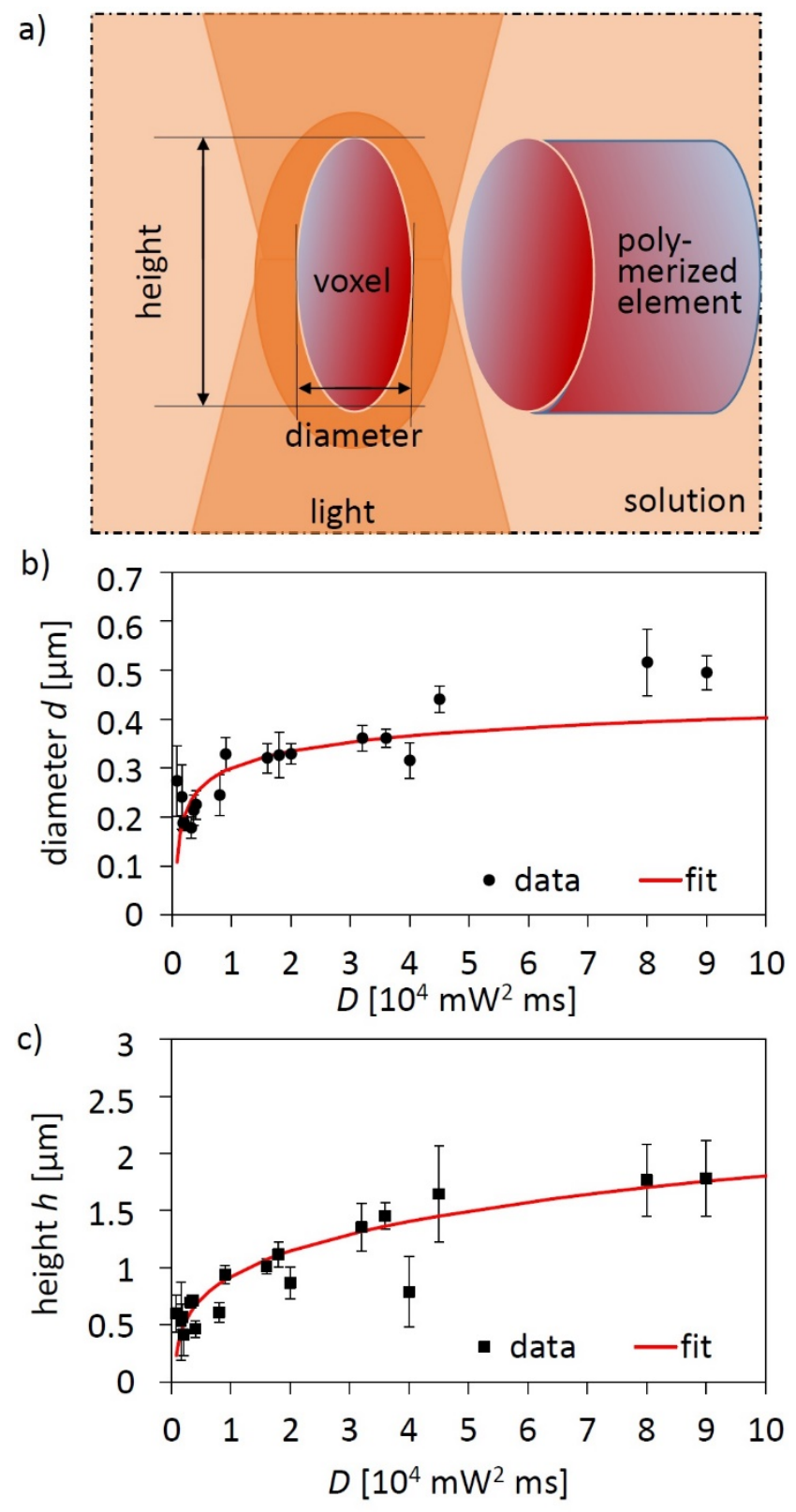

Figure 3 Characterization of BSA-FAD-RhB. a) Schematic illustration of how SEM data was measured to obtain diameter and height data pairs to describe voxel size. b,c) Diameter and height of voxels measured by SEM (filled symbols) and corresponding fitting result (red line) are shown for c) voxel diameter and d) voxel height. The data fit was obtained with $\chi^{2}=5.34$, and $\mathrm{R}^{2}=0.76$ for 18 data pairs $(\mathrm{n}=4)$.

Furthermore, the rapid fabrication is comparable to the three-component photosensitizing system BSA-FAD-RhB even though all components were reduced to $50 \%$ when introducing collagen in commercial buffer. Especially for cell adhesion promoting proteins, conventional 2D cell culture experiments showed that cell adhesion was observed for mixtures of low amounts of cell adhesion proteins and nonadhesion proteins such as BSA [20]. Therefore, the concept of three-component photosensitizing system complemented with a)
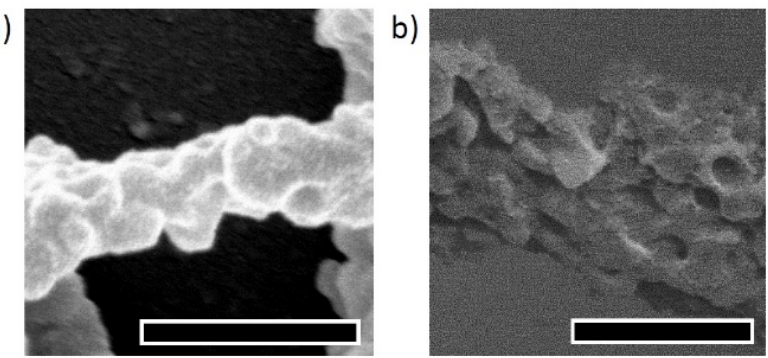

c)

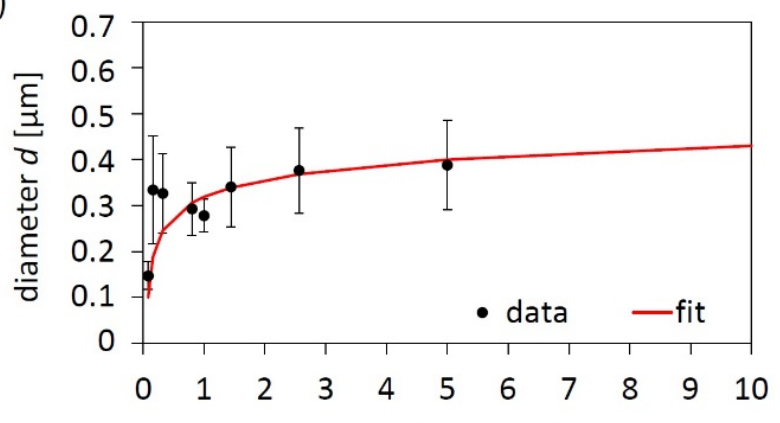

d)

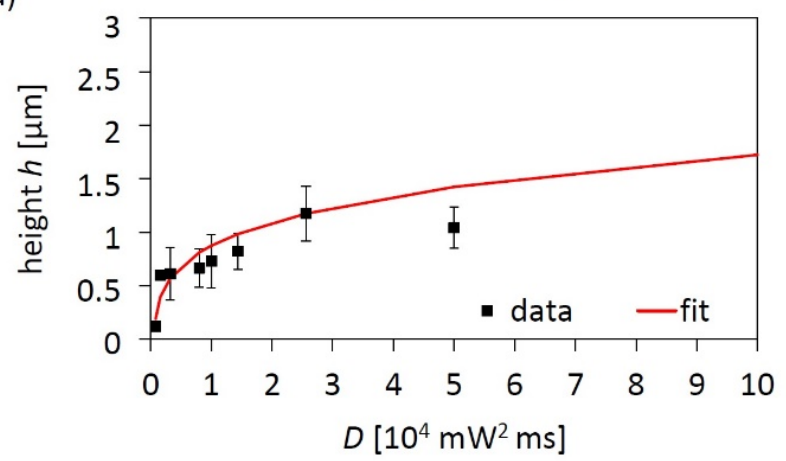

Figure 4 Characterization of BSA-FAD-RhB and collagen type-IV. Representative SEM images showing a) top view and b) side view of beam structure at $D=1600$. Scale bars represent $500 \mathrm{~nm}$. c,d) SEM measurements (filled symbols) of c) diameter and d) height respectively, and corresponding fitting result (red line) are shown for c) voxel diameter and d) voxel height. The data fit was obtained with $\chi^{2}=7.71$, and $\mathrm{R}^{2}=0.62$ for 8 data pairs $(\mathrm{n}=3)$.

low amounts of the protein of interest might be expected to become relevant for cell adhesion applications. Beyond protein binding affinity, other protein functions might also remain intact when proteins are complemented with a BSA-based photosensitizing system.

\subsection{Comparison of the described proteinaceous photosensitizing systems}

We summarize and compare the results of three different polymerization systems based on one polymerization model [11] (Figure 5, Table 1). Table 1 shows the different components of BSA-FAD, BSA-FAD-RhB and BSA-FAD$\mathrm{RhB}$ with collagen, highlighting the approximate changes in $\mathrm{z}-$ axis diffraction limit due to organic solvents influencing refractive 
Table 1 Summary of proteinaceous pre-polymer solutions

Comprehensive summary describing of conventional two-component system BSA-FAD [11], and three-component systems introduced in this paper, BSA-FAD-RhB and BSA-FAD-RhB with collagen. Protein and photosensitizer amounts are summarized regardless the number of components. Dehydration loss was evaluated by measuring beam length in liquid and after freeze-dry. The refractive index is estimated from [19] and $z_{\text {diff }}$ is derived (Appendix A). $r_{\text {diff }}$ is $0.34 \mu \mathrm{m}$ overall. Lastly, fitting results are displayed as scaling factors radial orientation, $\alpha_{R}$, and axial orientation, $\alpha_{Z}$, compressively describing complex kinetics of radical chain reaction and diffusion, and the threshold condition $D_{t h}$ derived from fitting parameter $F$.

\begin{tabular}{|c|c|c|c|}
\hline & $\begin{array}{c}\text { BSA- } \\
\text { FAD }[11]\end{array}$ & $\begin{array}{c}\text { BSA-F AD- } \\
\text { RhB }\end{array}$ & $\begin{array}{c}\text { BSA-FAD } \\
\text { RhB with } \\
\text { collagen }\end{array}$ \\
\hline $\begin{array}{c}\text { protein } \\
{[\mu \mathrm{M}]}\end{array}$ & 3030 & 760 & 380 \\
\hline $\begin{array}{l}\text { protein } \\
{[\mathrm{mg} / \mathrm{mL}]}\end{array}$ & 200 & 50 & 25.125 \\
\hline $\begin{array}{c}\text { sensitizer } \\
{[\mu \mathrm{M}]}\end{array}$ & 1000 & 860 & 430 \\
\hline $\begin{array}{l}\text { aqueous } \\
\text { fraction of } \\
\text { solution }\end{array}$ & $50 \%$ & $12.5 \%$ & $65.25 \%$ \\
\hline $\begin{array}{c}\text { organic } \\
\text { fraction of } \\
\text { solution }\end{array}$ & $50 \%$ & $87.5 \%$ & $34.75 \%$ \\
\hline $\begin{array}{l}\text { observed } \\
\text { dehydration } \\
\text { loss }\end{array}$ & $18 \% \pm 6 \%$ & $12 \% \pm 5 \%$ & $14 \% \pm 5 \%$ \\
\hline $\begin{array}{l}\text { estimated } \\
\text { refractive } \\
\text { index[18] }\end{array}$ & 1.4071 & 1.4619 & 1.3977 \\
\hline$z_{\text {diff }}[\mu \mathrm{m}]$ & 1.120 & 1.164 & 1.112 \\
\hline$\alpha_{R}$ [a.u.] & $0.61 \pm 0.04$ & $0.52 \pm 0.02$ & $0.56 \pm 0.04$ \\
\hline$\alpha_{Z}$ [a.u.] & $0.55 \pm 0.05$ & $0.44 \pm 0.02$ & $0.45 \pm 0.04$ \\
\hline$\alpha_{Z} / \alpha_{R}$ [a.u.] & $0.9 \pm 0.1$ & $0.85 \pm 0.05$ & $0.80 \pm 0.09$ \\
\hline $\begin{array}{c}D_{t h} \\
{\left[\mathrm{~mW}^{2} \mathrm{~ms}\right]}\end{array}$ & $2800 \pm 680$ & $550 \pm 70$ & $610 \pm 70$ \\
\hline
\end{tabular}

indices [19], fitting values and threshold condition in conjunction with protein and photosensitizer amounts. In comparison with BSA-FAD, BSA-FAD-RhB has reduced protein amount with comparable sensitizer amount and additionally higher organic solvent percentage, possibly influencing the photosensitizing system. BSA-FAD-RhB with collagen has reduced protein and photosensitizer amounts, and its fraction of organic solvent is comparable to BSA-FAD.
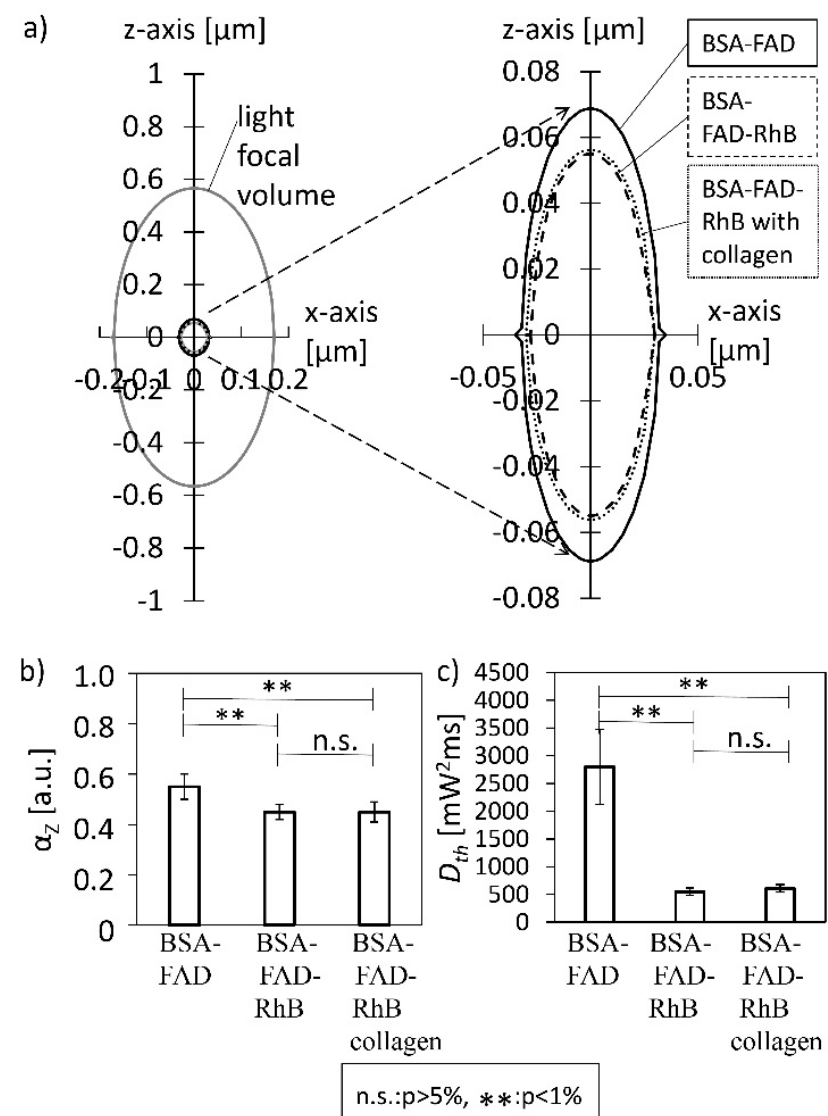

Figure 5 Comparison of the described proteinaceous photosensitizing systems. a) Illustration of polymerized voxel sizes for different photosensitizing systems: Cross section of the focal volume of applied light is shown with $r_{\text {diff }}=0.34 \mu \mathrm{m}$ and $z_{\text {diff }}=1.132 \mu \mathrm{m}$. With $D / D_{t h}=1.1$ and respective scaling factors the resulting cross sections of polymerized voxels are shown for BSA-FAD (black), BSA-FAD-RhB (dashed) and BSA-FAD-RhB with collagen (dotted). b,c) Summarizing representation of fitting results for b) $\alpha_{Z}$ and $D_{t h}$ (Table 1) with indication of significance $(* *, \quad \mathrm{p}<1 \%)$ and nonsignificance (n.s.).

In order to visualize the implication of different scaling factors to fabrication sizes, a laser focal volume of $r_{\text {diff }}=0.34 \mu \mathrm{m}$ and $z_{\text {diff }}=1.132 \mu \mathrm{m}$ is assumed and scaling factors are applied with a near-fabrication-threshold condition $D / D_{t h}=1.1$ (Figure $5 \mathrm{a}$ ). The illustration shows that with regards to effective polymerized voxel shape, BSA-FAD-RhB and BSA-FAD-RhB with collagen are similar against the significantly different ratio of BSA-FAD (Figure $5 \mathrm{~b}$ ). This result illustrates that despite different organic solvents and material amounts, the introduction of a third component, RhB, affected the polymerization kinetics and thereby polymerization volume.

It has been shown that polarization effects polymerization via absorption coefficient, ionization rate and heat conduction [22]. We obtained all experimental data for the same type of linear horizontal polarization and the same fabrication orientation by reuse of coordinate data files. 
Changing the angle between polarization and fabrication direction or the type of polarization, height-to-width ratio of the voxel shape might change up to $12 \%$ [22]. As heat conduction and material diffusion play a role in the height-towidth ratio, the exact percentage might be different for each of the discussed photosensitizing systems due to different solvents.

As previously discussed, BSA-FAD-RhB and BSA-FAD$\mathrm{RhB}$ with collagen have a considerably lower threshold condition, $D_{t h}$ (Figure $5 \mathrm{c}$ ), enabling faster fabrication (Table 1). This similarity between BSA-FAD-RhB and BSA-FAD$\mathrm{RhB}$ with collagen suggests that a higher organic solvent fraction as in BSA-FAD-RhB might not be relevant to lowering fabrication thresholds. It might be possible that $\mathrm{pH}$ counteracts solvent effects in these photosensitizing systems; a large fraction of collagen type-IV solution tethered at $\mathrm{pH}=3$ was used in BSA-FAD-RhB with collagen, instead of $\mathrm{pH}=7$ buffer used in BSA-FAD and BSA-FAD-RhB.

It might be worth noting that despite the reduction in protein amount, the dehydration loss was less (Table 1) when applying a three-component photosensitizing system. The density of the formed cross-links might be comparable within the here presented photosensitizing systems; the availability of photosensitizers might lead to a more efficient embedding of protein radicals into the proteinaceous microstructure.

\section{Conclusion}

In this paper, we showed that a proteinaceous threecomponent photosensitizing system due to the introduction of an additional sensitizer enables desirable increase of fabrication speed simultaneously with desirable reduction of material amounts. The results do not equal record setting properties of synthetic materials [21], but advance recent protein cross-linking $[8,9,11,16]$. Thereby, arrays of protein microstructures were fabricated within reasonable fabrication periods of minutes to hours. A previously introduced polymerization model [11] enables feature size prediction and fabrication threshold conditions analysis for three-component photosensitizing systems.

Furthermore, we showed that a low amount of a protein of interest such as collagen type-IV was added to the introduced three-component photosensitizing system and enabled rapid microfabrication. Therefore, we envision three-component photosensitizing and photoinitiating systems with low amounts of functional proteins to become an important fabrication tool for proteinaceous microdevices.

\section{Acknowledgement}

This work was partly supported by the Takeuchi Biohybrid Innovation Project, Exploratory Research for Advanced Technology (ERATO), Japan Science and Technology (JST), Japan. D.S. received a scholarship from the Ministry of Education, Culture, Sports, Science, and Technology of Japan.

\section{Appendix: Protein polymerization model [11]}

$$
d(D)=\alpha_{R} r_{\text {diff }} \sqrt{\ln \left(F \frac{1}{r_{\text {diff }}^{4}} D\right)}
$$

$$
\begin{aligned}
& h(D)=\alpha_{Z} z_{\text {diff }} \sqrt{\sqrt{F \frac{1}{r_{\text {diff }}^{4} D}-1},} \\
& r_{\text {diff }}=\frac{0.61 \lambda}{N . A .}, z_{\text {diff }}=\frac{2 \lambda n}{N \cdot .^{2}}
\end{aligned}
$$

where $D$ is the dose equivalent dependent on laser power $P$ and exposure time $t$ by $D(P, t)=P^{2} t . F$ is a factor summarizing parameters for laser light, two-photon absorption and pre-polymer solution [11] with the relationship to the threshold dose equivalent where $d=h=0$ of $D_{t h}=F / r_{\text {diff }}^{4} \cdot r_{\text {diff }}$ and $z_{\text {diff }}$ are the diffraction limits describing the laser beam as approximated Gaussian laser beam in dependency of wavelength $\lambda$, numerical aperture N.A. and refractive index $n$. Scaling factors in radial orientation, $\alpha_{R}$ and axial orientation, $\alpha_{Z}$, compressively describe complex kinetics of radical chain reaction and diffusion.

\section{References}

[1] J. Fischer, and M. Wegener: Laser Photonics Rev., 7, (2013) 22.

[2] T. Bückmann, M. Thiel, M. Kadic, R. Schittny, and M. Wegener: Nat. Commun., 5, (2014) 4130.

[3] J.D. Spikes, H.-R. Shen, P. Kopečková, and J. Kopeček: Photochem. Photobiol., 70, (1999) 130.

[4] J.D. Pitts, P.J. Campagnola, G.A. Epling, and G.A. Goodman: Macromolecules, 33, (2000) 1514.

[5] Y.-L. Sun, W.-F. Dong, R.-Z. Yang, X. Meng, L. Zhang, Q.-D. Chen, and H.-B. Sun: Angew. Chem. Int. Ed., 51, (2012) 1558.

[6] M.R. Lee, I.Y. Phang, Y. Cui, Y.H. Lee, and X.Y. Ling: Small, 11, (2015) 740.

[7] C.L. Lay, M.R. Lee, H.K. Lee, I.Y. Phang, and X.Y. Ling: ACS Nano, 9, (2015) 9708.

[8] Y.-L. Sun, S.M. Sun, B.Y. Zheng, Z.H. Hou, P. Wang, X.L. Zhang, W.-F. Dong, L. Zhang, Q.-D. Chen, L.M. Tong, and H.-B. Sun: IEEE Photon. Technol. Lett., 28, (2016) 629.

[9] B. Kaehr, and J.B. Shear: Proc. Natl. Acad. Sci. U. S. A., 105, (2008) 8850.

[10] C.-L. Lin, M.-J. Pan, H.-W. Chen, C.-K. Lin, C.-F. Lin, and P.L. Baldeck: Proc. SPIE, 9310, (2015) 93100D.

[11] D. Serien, and S. Takeuchi: Appl. Phys. Lett., 107, (2015) 013702.

[12] J.P. Fouassier, X. Allonas, and D. Burget: Prog. Org. Coat., 47, (2003) 16.

[13] J.P. Fouassier, and J. Lalevée: RSC Adv., 2, (2012) 2621.

[14] P. Bartasun, H. Cieśliński, A. Bujacz, A. Wierzbicka-Woś, and J. Kur: PLOS ONE, 8, (2013) e55697.

[15] Y. Bi, and D.C. Neckers: Macromolecules, 27, (1994) 3683.

[16] B. Kaehr, R. Allen, D.J. Javier, J. Currie, and J.B. Shear: Proc. Natl. Acad. Sci. U. S. A., 101, (2004) 16104.

[17] C.E. Aitken, R.A. Marshall, and J.D. Puglisi: Biophys. J., 94, (2008) 1826.

[18] A. LeGall, D. Dulin, G. Clavier, R. Meallet-Renault, P. Bouyer, K. Perronet, and N. Westbrook: ChemPhysChem, 12, (2011) 1657.

[19] R.G. LeBel, and D.A.I. Goring: J. Chem. Eng. Data, 7, (1962) 100.

[20] J.E. Koblinski, M. Wu, B. Demeler, K. Jacob, and H.K. Kleinman: J. Cell Sci., 118, (2005) 2965.

[21] M. Malinauskas, A. Žukauskas, S. Hasegawa, Y. Hayasaki, V. Mizeikis, R. Buividas, and S. Juodkazis: Light Sci. Appl., 5, (2016) e16133.

[22] S. Rekštytè, T. Jonavičius, D. Gailevičius, M. Malinauskas, V. Mizeikis, E.G. Gamaly, and S. Juodkazis: Adv. Opt. Mater., 4, (2016) 1209.

(Received: June30, 2016, Accepted: July18, 2017) 